\title{
Palisaded Neutrophilic Granulomatous Dermatoses: An Unusual Lesion Affecting the Palms
}

JASON W. AN, MD, Internal Medicine, University of Alberta, Edmonton, Alberta; MOHAMMAD BARDI, MD, Rheumatology, University of British Columbia, British Columbia, Canada. Address correspondence to Dr. J.W. An, 8210-111st NW Edmonton, Alberta T6G 2C7, Canada.

E-mail: Jason.internalmed@gmail.com. Written consent has been provided by the patient for her case to be published anonymously. Ethics board approval was not required in accordance with the policies of the authors' institutions. J Rheumatol 2018;45:1706-7; doi:10.3899/jrheum.180121

Palisaded neutrophilic granulomatous dermatoses (PNGD) is a spectrum of histologic diagnosis featuring abnormal collagen deposition, palisaded histiocytes, and small granulomas in the absence of leukocytoclastic vasculitis ${ }^{1}$. It is thought to reflect immune complex deposition and is associated with rheumatoid arthritis, connective tissue diseases, hematologic disorders, infections, and medications such as tumor necrosis factor- $\alpha$ inhibitors ${ }^{2}$ (Figure 1).

A case of PNGD that was eventually confirmed by biopsy involved a 36-year-old female with mixed connective tissue disease who presented with an exacerbation of gastroparesis and lesions on the hands and elbows.

Acutely, her vomiting resolved with symptomatic treatment. Her hospital stay thereafter was marked by nodular, painful lesions lasting 3 to 5 days on the fingertips, palms, and elbows (Figure 2). Examination of the nails and feet were normal. Swabs of her lesions yielded staphylococcus, and she was treated with antibiotics with minimal improvement. An echocardiogram excluded vegetation, and blood cultures were negative. Complement levels were normal. Biopsy of an active lesion ultimately revealed PNGD.
PNGD often affects the elbows, trunk, and hands. Palmar involvement occurs, and thus PNGD should be considered alongside differential diagnoses such as Osler nodes, secondary syphilis, erythema multiforme, and lichen planus in the appropriate clinical context. Clinical course varies from spontaneous resolution to persistence despite therapy. Treatment includes dapsone, topical or systemic corticosteroids, and management of the underlying disorder ${ }^{2}$. Our patient was treated with high-dose topical corticosteroids with resolution of lesions.

\section{REFERENCES}

1. Rosenbach M, English JC 3rd. Reactive granulomatous dermatitis: a review of palisaded neutrophilic and granulomatous dermatitis, interstitial granulomatous dermatitis, interstitial granulomatous drug reaction, and a proposed reclassification. Dermatol Clin 2015;33:373-87.

2. Gulati A, Paige D, Yaqoob M, Proby CM, Cerio R, Harwood CA. Palisaded neutrophilic granulomatous dermatitis associated with systemic lupus erythematosus presenting with the burning rope sign. J Am Acad Dermatol 2009;61:711-4. 


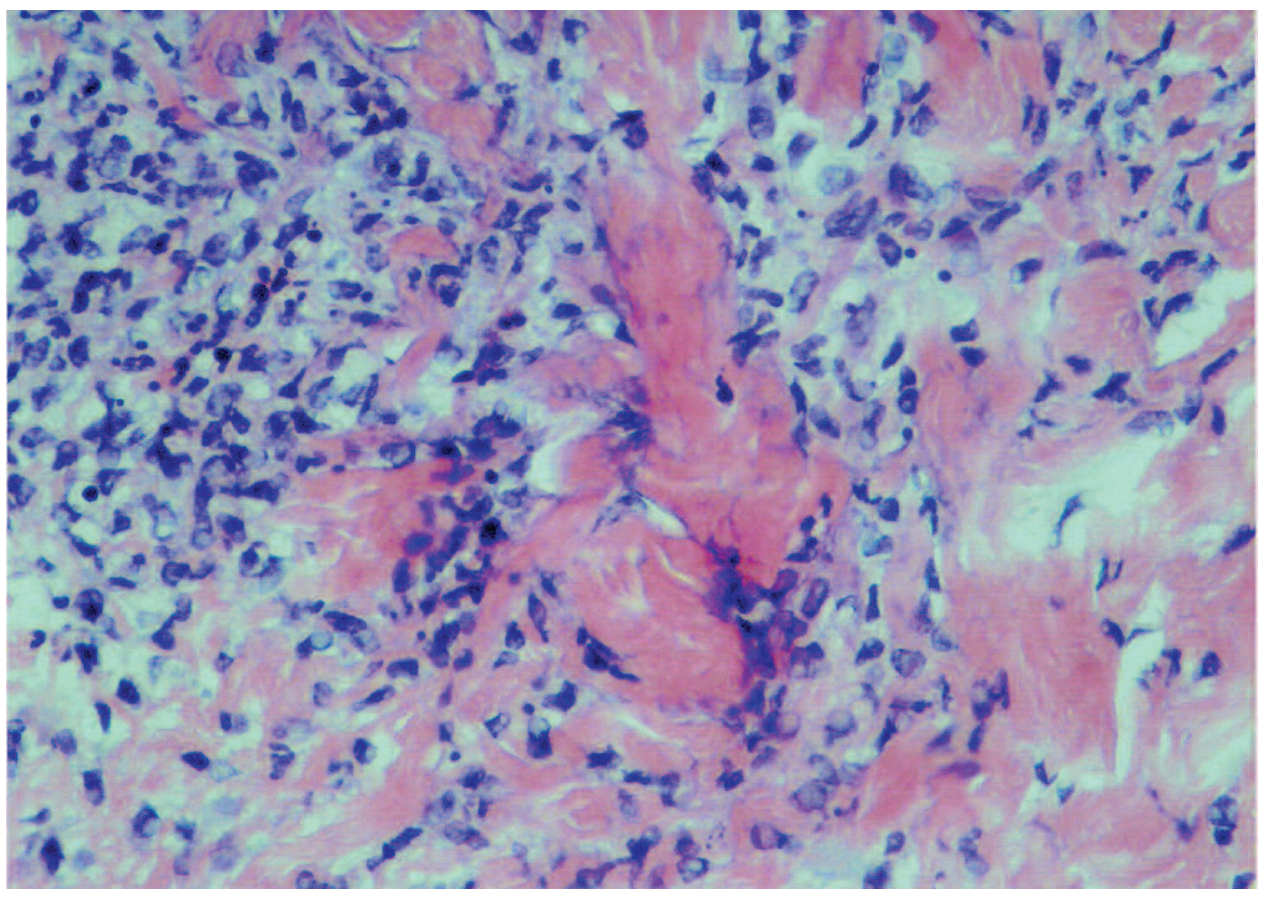

Figure 1. Degenerated collagen bundles encircled by a histiocytic infiltrate with nuclear debris, $400 \times$ stained with $\mathrm{H} \& \mathrm{E}$.

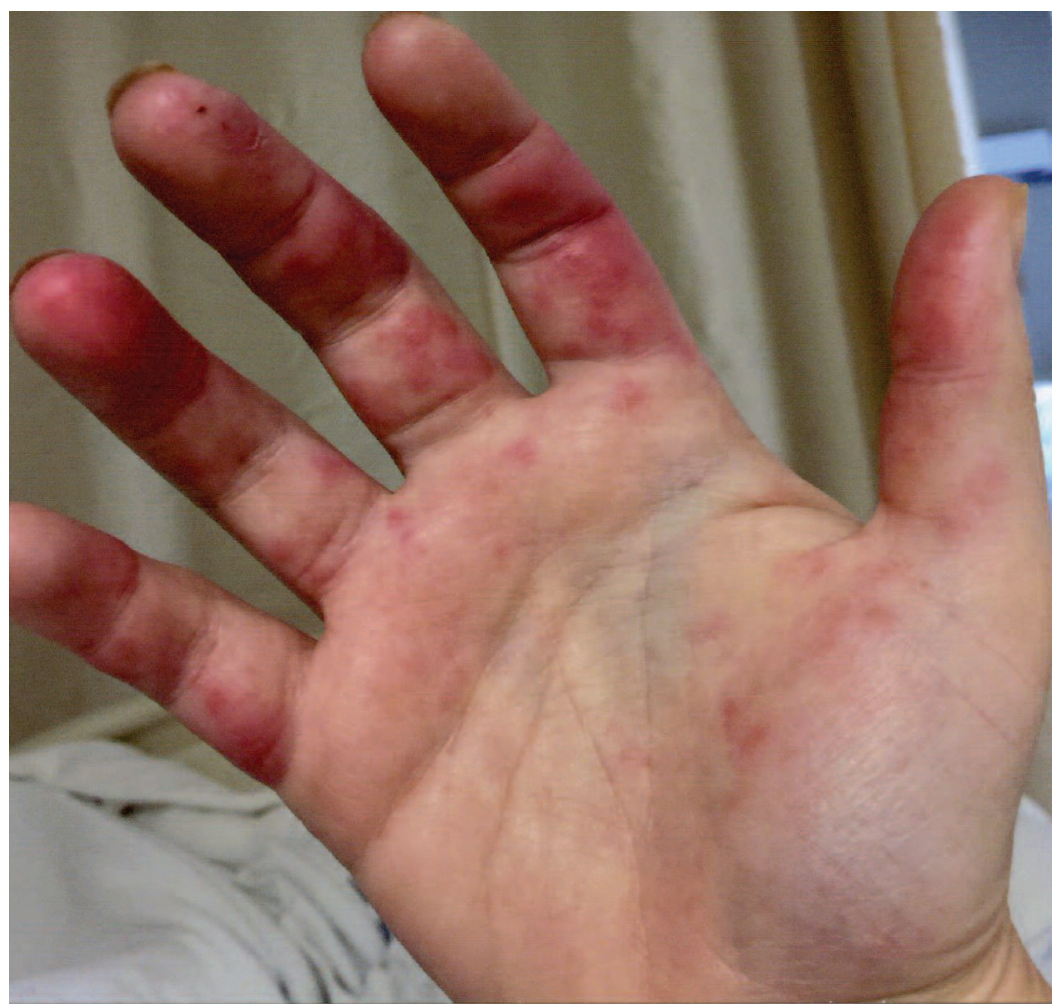

Figure 2. Erythematous nodular painful palmar lesions. 